Helgoländer wiss. Meeresunters. 24, 327-332 (1973)

\title{
Observations sur le metabolisme d'un poisson veneneux des eaux tropicales: Ctenochaetus striatus
}

\author{
G. Cuzon, R. Bagnis \& J. Lagraulet \\ Institut de Recherches Médicales Lonis Malardé; Papeete, Tabiti
}

\begin{abstract}
Observations on the metabolism of a poisonous fish from tropical waters: Ctenochaetus striatus. Data on epidemiology and ecology of toxic and non-toxic fish are presented. C. striatus is one of the first links of the lagoon food chain. The degree of toxicity was determined by feeding fish muscles to cats over a period of 72 hours. The most significant differences between poisonous and non-poisonous C. striatus were observed in regard to water content of muscles: poisonous fish contained 3 to $4 \%$ more water that non-poisonous fish. Protein and lipid contents of poisonous fish were comparable to those of non-poisonous representatives. Ions in the blood serum varied according to the presence or absence of toxin in the fish.
\end{abstract}

\section{INTRODUCTION}

Dans les eaux tropicales, il existe environ 500 familles de poissons dont la chair peut-être vénéneuse. La principale toxine responsable des intoxications alimentaires par ingestion de ces poissons est la ciguatoxine, caractérisée par SCHEUER et al. (1967). A lorigine elle serait produite par un organisme benthique, peut-être une algue. Les poissons herbivores représentent un des premiers maillons d'une chaîne alimentaire au cours de laquelle la toxine se concentre. Ainsi les poissons carnivores donnent lieu à des empoisonnements particulièrement graves dans certains cas (BAGNIs 1968). Le «poisson-chirurgien» Ctenochaetus striatus semble jouer un tôle important dans cette chaîne alimentaire liée à la toxine (HELFRICH \& BANNER 1963). Des études ont été réalisées sur un composé liposoluble isolé d'Acanthuridae (BANNER 1967), et sur une substance hydrosoluble (Hashimoto et al. 1969). Quelques données écologiques ont été rapportées sur le poisson-chirurgien afin de mieux caractériser son biotope. Il n'existe pas d'études sur le métabolisme de C. striatus et la comparaison entre des spécimens inoffensifs et des spécimens toxiques peut permettre une meilleure compréhension des problèmes posés par l'origine biologique de la toxine. Les poissons sont capturés au fusil sous-marin, par lots de vingt individus, dans la baie de Popote (Fig. 1), à proximité d'une passe du lagon de Tahiti, et à Punaauia. Les dissections ont lieu sur place aussitôt après la capture. Un carré de chair est découpé sur le côté droit au dessous de la ligne latérale près de l'épine caudale. Ce fragment est placé dans une coupelle et conservé à $-30^{\circ} \mathrm{C}$. Après pesée, les échantillons sont analysés. 
La toxicité est contrôlée en donnant $10 \%$ de leur poids en chair de poisson à de jeunes chats mis à jeûn 48 heures avant le test. L'échelle de toxicité adoptée va de $0 \dot{a}+5$. Lorsqu'aucun signe de faiblesse n'est décelé chez le chat, le poisson est noté 0 . L'indice +5 correspondant à un poisson qui entraîne la mort du chat. +1 à +4 sont des indices intermédiaires pour des réactions anormales (vomissements, faiblesse générale) du chat après 72 heures d'expérience. D'une façon générale le nombre de poissons toxiques est toujours inférieur au nombre de poissons inoffensifs dans un même lot.

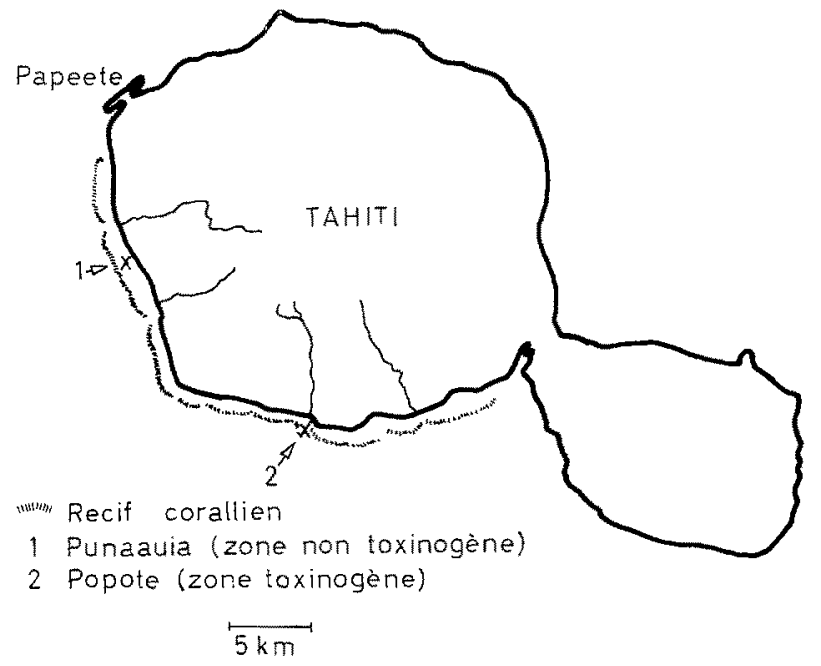

Fig. 1: Carte des lieux de pêche de Ctenochatus striatus

La teneur en eau est obtenue en desséchant chaque échantillon pendant 12 heures $\grave{a}+80^{\circ} \mathrm{C}$ et en faisant refroidir à l'exsicateur en présence de silicagel. Le muscle sec est placé ensuite dans des extracteurs de Soxhlet. Le mélange chloroforme-méthanol en proportion $1 / 2$ est porté à ébullition pendant 5-6 heures et recueille les lipides musculaires. On évapore le solvant et les lipides restent au fond du ballon. Le calcul du pourcentage de lipides est rapporté au poids sec. Enfin, le muscle délipidé, desséché à nouveau est minéralisé dans de l'acide sulfurique en présence d'un catalyseur à base de sélénium. Le dosage de l'azote total est effectué dans un appareil semi-automatique de Kjeldhal. La quantité d'azote total obtenu multipliée par le coefficient universel 6,25 fournit une bonne approximation de la teneur en protéines dans le muscle. Le sang des poissons prélevé juste après la capture est recueilli dans des tubes à hémolyse. Le sérum est analysé par photométrie de flamme pour obtenir les teneurs en $\mathrm{Na}^{+}$et $\mathrm{K}^{+}$, par la technique de FoLIN pour les protéines totales.

\section{RESULTATS ET DISCUSSION}

En moyenne, les analyses portent sur deux grammes de muscle frais. Les poissons inoffensifs contiennent moins d'eau $(\mathrm{m}=78 \%$ ) que les poissons vénéneux $(\mathrm{m}=81 \%)$. 
Si l'on prend deux lots de poissons de même poids (environ $110 \mathrm{~g}$ ) en dehors de la période de reproduction, la différence est significative $(P=0,05)$ pour un premier lot de 20 individus et très significative $(P=0,02)$ pour un autre lot de 20 unités. Il est à remarquer parmi les poissons «toxiques» deux catégories (Fig. 2), les poissons faiblement toxiques $(+1,+2), \mathrm{n}=13$ et les poissons fortement toxiques $(+4,+5), \mathrm{n}=13$.

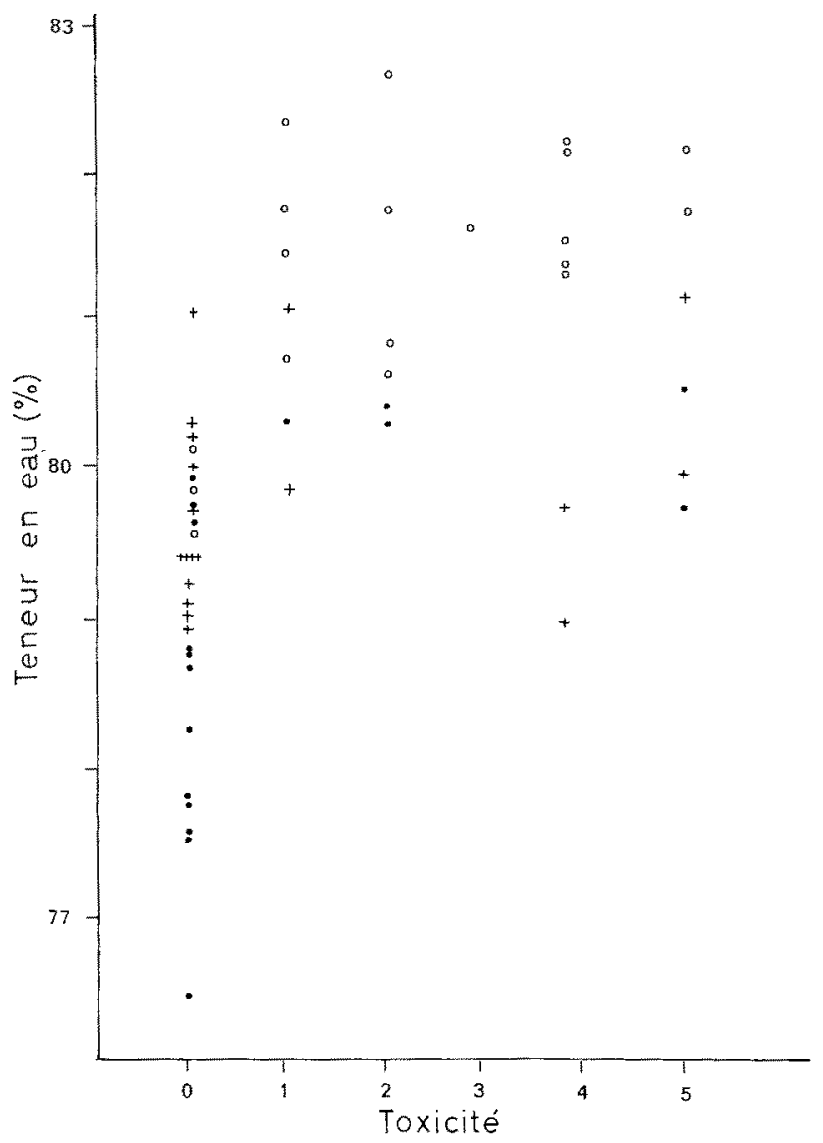

Fig. 2: Teneur en eau du muscle en fonction de la toxicité de poissons-chirurgiens de la zone de Popote. (Les signes, 0 , + et - distinguent les trois captures effectuées à 1 semaine d'intervalle)

La catégorie +3 est le plus souvent difficile à définir. La teneur en eau d'un poisson est une donnée extrêmement variable; Soudan et al. (1965) indiquent des valeurs de 57 à $65 \%$ chez un même individu en différents points le long de la ligne latérale. Les prélèvements doivent être effectués de façon très rigoureuse. Les individus étant de même poids, il est cependant nécessaire de faire un test d'homogénéité qui ne montre aucune différence due au sexe. Toutes choses étant égales par ailleurs, l'eau du muscle varie dans les marges indiquées suivant la présence ou l'absence de toxine chez C. striatus. Il est permis de penser alors que la fraction toxique hydrosoluble (Hashimoto et al. 1969) est prépondérante au niveau du muscle. 
L'analyse des lipides totaux ne montre pas de différence significative $(P=0,50)$ entre les poissons inoffensifs et les toxiques. Cependant on peut distinguer le groupe 0 toujours inférieur à $12 \%$ (Fig. 3), le groupe $(+1+2$ ) autour de $12,5 \%$ et le groupe $(+4,+5)$ légèrement supérieur à la moyenne avec $12,9 \%$. L'expérience porte sur un trop petit nombre d'individus $(\mathrm{n}=20)$, pour parler d'une augmentation de la quantité de graisses chez les poissons ciguatoxiques. La toxine existe sous forme de lipide,

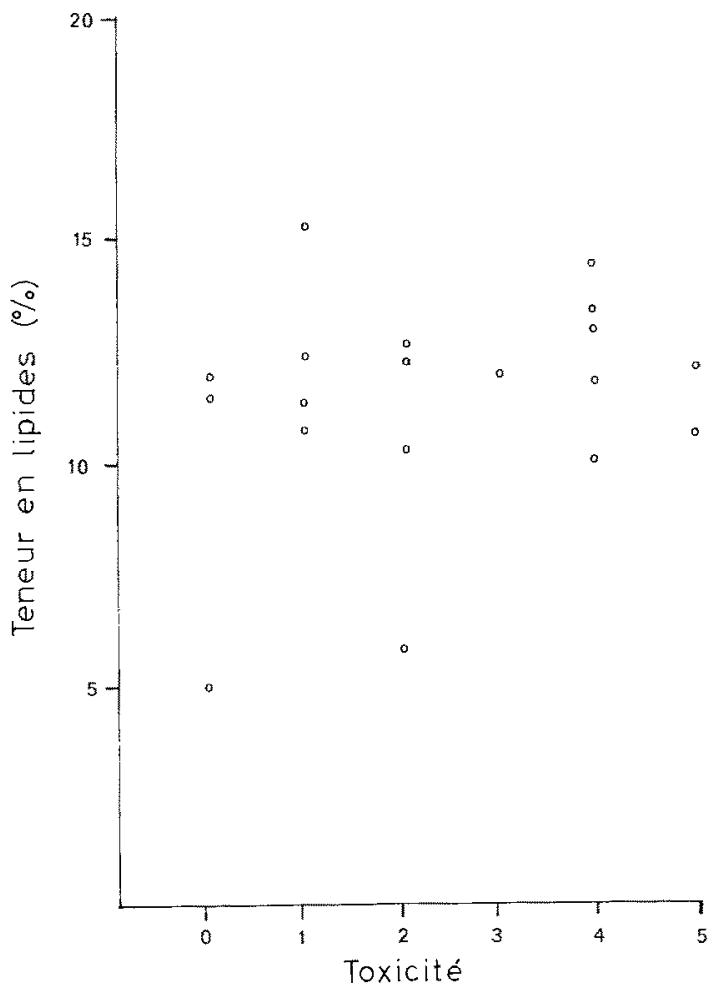

Fig. 3: Teneur en lipides du muscle sec de Ctenochaetus striatus en fonction de la toxicité de la chair (zone de Popote)

appelée ciguatoxine (ScHEUER et al. 1969). Nous avons pu vérifier que la technique d'extraction utilisée donnait un mélange de lipides chromatographiquement semblable au résultat de l'extraction par la méthode de Scheuer. Il est probable que la quantité de toxine extraite au moyen du Soxhlet ne soit pas mise en évidence pondéralement.

L'azote du muscle de C. striatus dosé à partir de 20 échantillons comprenant 2 catégories (toxique ou sain) donne des résultats significativement différents $(P=0,05)$, $\mathrm{m}=13,6 \mathrm{~g}$. p. $100 \mathrm{~g}$ de poids sec pour des poissons toxiques, $\mathrm{m}=13,9 \mathrm{~g} . \mathrm{p} .100 \mathrm{~g}$ chez des poissons non toxiques. La même valeur, 13,9 p. $100 \mathrm{~g}$ est retrouvée en analysant 20 poissons de la zone 1 réputée non toxinogène.

Cette baisse de protéines n'est plus significative au niveau des protéines sériques des poissons puisque $\mathrm{m} 1=13,4 \mathrm{~g} / 1$ pour des spécimens toxiques et $\mathrm{m} 2=12,9 \mathrm{~g} / 1$ pour 
les autres $(P=0,50)$. Au cours d'un précédent travail (Cuzon et al. 1972) il a été mis en évidence électrophorétiquement des fractions surnuméraires lentes type gammaglobulines dans un grand nombre de sérums de poissons toxiques. Cette différence semble-t-il n'est pas définissable quantitativement.

Quelques données sont rapportées au tableau I relatives aux quantités de $\mathrm{K}^{*}$ et de $\mathrm{Na}^{+}$trouvées dans le sérum des poissons inoffensifs ou toxiques. On remarque la

\section{Tableau 1}

Valeurs moyennes en milliéquivalents par litre des quantités de sodium et de potassium présentes dans le sérum des poissons-chirurgiens de la zone 2 (s: écart type). Les comparaisons de moyenne sont faites à l'aide du test $\mathrm{t}$ de STUDENT-FISHER

\begin{tabular}{|cccc|}
\hline Cations & $\begin{array}{c}\text { Poissons } \\
\text { non toxiques } \\
(\mathrm{n}=14)\end{array}$ & $\begin{array}{c}\text { Poissons toxiques } \\
(\mathrm{n}=10)\end{array}$ & $\begin{array}{c}\text { Différence } \\
\text { significative }\end{array}$ \\
\hline $\mathrm{Na}^{+}$ & $\begin{array}{c}240 \\
(\mathrm{~s}=24)\end{array}$ & $\begin{array}{c}211 \\
(\mathrm{~s}=73)\end{array}$ & $\mathrm{P}>0,01$ \\
$\mathrm{~K}^{+}$ & $\begin{array}{l}5,4 \\
(\mathrm{~s}=2,5)\end{array}$ & $\begin{array}{c}8,9 \\
(\mathrm{~s}=4,3)\end{array}$ & $\mathrm{P}>0,02$ \\
\hline
\end{tabular}

diminution du sodium et l'augmentation du potassium présents dans le sérum. Toutefois, peut-être en raison du faible nombre d'analyses il n'existe pas de corrélation entre les deux variables. Cette modification éventuelle de la balance ionique doit être vérifée au niveau des autres ions, chlore, magnésium, calcium, notamment.

\section{CONCLUSION}

Il est cerrain que le petit nombre d'analyses effectuées ne permet pas de tirer des conclusions définitives, quoique les tests statistiques employés pour de faibles échantillons restent valables. Les tests d'homogénéité ne montrent aucune différence due au sexe ou au poids des individus.

Les quelques observations recueillies montrent des variations au niveau du muscle et du sérum d'un poisson inoffensif à un poisson toxique. Les différences les plus intéressantes concernent la teneur en eau du muscle: elle varie suivant la présence ou l'absence de toxine chez le poisson. Nous nous proposons d'étudier plus précisément les acides gras contenus dans la fraction lipide au moyen de la chromatographie gazeuse. Il serait également utile de connaître la nature précise des protéines qui entrent dans la composition du muscle en fonction de la présence ou de l'absence de toxine.

En raison des variations de la teneur en eau certaines mesures de résistivité par exemple sont à retenir pour éventuellement détecter des espèces toxiques. Compte tenu de l'expansion et du développement des pêches et de l'aquaculture en zone tropicale, il devient nécessaire de pouvoir contrôler l'état physiologique des animaux en élevage. 


\section{RESUME}

1. La toxicité des poissons est contrôlée en faisant absorber $10 \%$ de leur poids en chait de poisson à de jeunes chats.

2. Les analyses de la teneur en eau du muscle des poissons montrent une augmentation significative chez les poissons ciguatérigènes. La fraction toxique hydrosoluble apparaît donc prépondérante sur la fraction liposoluble (ciguatoxine).

3. L'azote total du muscle montre de légères variations suivant qu'on s'adresse à un poisson inoffensif ou toxique. La teneur en lipides musculaires est relativement constante.

4. Les protéines totales du sérum ont des valeurs voisines $m 1=13,4 \mathrm{~g} / 1$ chez les toxiques, $\mathrm{m} 2=12,9 \mathrm{~g} / \mathrm{l}$ chez les non toxiques $(\mathrm{P}=0,50)$.

5. Les ions $\mathrm{Na}^{+}$et $\mathrm{K}^{+}$du sérum sont dosés au photomètre à flamme. Chez les poissons toxiques il y a augmentation du $\mathrm{K}^{+}$et diminution du $\mathrm{Na}^{+}$.

\section{LITTERATURE CITEE}

Bagnis, R., 1968. Clinical aspects of ciguatera (Fish poisoning) in French Polynesia. Hawaii med. J. 28, 25.

BANner, A. H., 1967. Marine toxins from the Pacific. I. Advances in the investigation of fish toxins. In: Animal Toxins. Ed. by F. E. Russell \& P. R. SAunders. Pergamon Press, Oxford, $157 \mathrm{pp}$.

Cuzon, G., Bagnis, R., Moreau, J. P. \& Lagraulet, J., 1972. Les protéines sériques de C. striatus: modifications du schéma électrophorétique chez les poissons ciguatérigènes. (à parâitre).

Hismmoto, Y., Yasumoto, T., Kamiya, H. \& Yoshida, T., 1969. Occurence of ciguatoxin and ciguaterin in ciguatoxic fishes in the Ryukyu and Amami Island. Bull. Jap. Soc. scient. Fish. 35, 327.

HiLFRICH, P. \& BANNER, A. H., 1963. Experimental induction of ciguatera toxicity in fish through diet. Nature, Lond. 197, 1025-1026.

Scheuer, P. J., Takahashi, W., Tsutsumi, J. \& Yoshida, T., 1967. Ciguatoxin isolation and chemical nature. Science, $N_{*} Y .155,1267-1268$.

Soudan, F., Anquez, M. \& Benezit, A., 1965. La conservation par le froid des poissons, crustacés et mollusques. Encyclopédie du froid. Baillière, Paris, 516 pp.

L'adresse de l'auteur premier: Dr. G. Cuzon

I. R. M. L. M.

Papeete, Tahiti

French Polynesia 